\title{
Review of: "Prevalence and Antimicrobial Susceptibility Pattern of Urinary Tract Infection among Pregnant Women Attending Hargeisa Group Hospital, Hargeisa, Somaliland"
}

Krzysztof Skowron, Joanna Kwiecińska-Piróg

1 Nicolaus Copernicus University

Potential competing interests: The author(s) declared that no potential competing interests exist.

The article we reviewed deals with a significant clinical problem. Quite a large group of patients was included in the study. The concept and layout of the work are correct and the article is written in the correct language. The work, however, leaves a certain dissatisfaction with regard to the information it could provide. For example, there are no data on the virulence of the tested microorganisms, as well as their genetic characteristics (assessment of genetic similarity, detection of antibiotic resistance genes and virulence genes).

Moreover, there are some specific concerns with this study, which include the following:

1. It is not clear that the clean-catch urine samples were collected as first morning sample or at least 4 hours after last urination. It is very important to properly prepare and correctly collect the urine sample, especially if asymptomatic urinary tract infection might be recognized on the base of obtained results. The hygiene before the sample collecting reduce the risk of false positive results in 20\% [Delanghe J, Speeckaert M. Preanalytical requirements of urinalysis. Biochem Med (Zagreb). 2014;24(1):89-104. Published 2014 Feb 15. doi:10.11613/BM.2014.011].

2. The methodology of antimicrobial susceptibility testing (AST) is based on the basic researches of Bauer (1966). Why authors did not use recommended by CLSI M02 most convenient method, cited below: "1. Prepare the inoculum by making a direct broth or saline suspension of isolated colonies selected from an 18- to 24-hour agar plate (use a nonselective medium, such as blood agar). 2. Adjust the suspension to achieve a turbidity equivalent to a 0.5 McFarland standard." Instead of this they used the alternative method - growth method, that was related with necessarily to do the additional broth culture. We are sure also that this method is recommended for Staphylyoccocus spp., because in CLSI M02-11 paper Stahylococcus spp. strains were excluded from this method.

3. Antibiotics included to the examination. In our opinion, in article about UTI should be included antimicrobials used only in UTI treatment. For this reason, I do no know, why authors tested susceptibility of Staphylococcus spp. to erythromycin and tetracyclins, and in Gram negative rods - to nalidixic acid, and tetracyclins. Beside, if all examination were done in pregnancy women, antimicrobials not recommended in this group should be also excluded (fluoroquinolones, aminoglycosides).

4. Interpretation of AST results. Authors interpreted all results according to CLSI 2020. In this document (and any else that we know) are no information about interpretation of zone inhibiotion around disc containing penicillin (10 $\mu \mathrm{g})$ or augmentin $(20 / 10 \mu \mathrm{g})$ - despite augmentin is a trade name of amoxicillin and clavulonic acid. According to what 
interpretation of mentioned above antimicrobials was done?

5. Authors included a lot of intrinsic resistance to results analysis, i.e.:Klebsiella pneumoniae, Citrobacterspp., Morganella morganii to ampicillin, Pseudomoans aeruginosa and Staphylococcus spp. to ceftriaxone. Authors skipped also information about ampC resistance that can interfere in susceptibility to aminopenicillins and cephalosporins I-III generation. We believe that it can led to changes in conclusions. Authors should take all this notes and to do new analysis of AST results.

6. According to description below the Table 5 MDR means resistance for three or more antibiotics. According to most common used definition, MDR means resistance to three or more antibiotics from various groups. The MDR definition should be clarify.

7. We believe that the use of a smaller number of age ranges (Table 6) could allow us to find some statistically significant relation. 